UDK $577.1: 61$

ISSN 1452-8258

J Med Biochem 38: 418-426, 2019

\title{
CORRELATION OF FGF23 AND BALP WITH BONE MINERAL DENSITY IN HEMODIALYSIS PATIENTS
}

\author{
KORELACIJA FGF23 I BALP SA MINERALNOM GUSTINOM KOSTIJU \\ KOD PACIJENATA NA HEMODIJALIZI
}

\author{
Mouna Bouksila ${ }^{1}$, Mehdi Mrad ${ }^{2}$, Wajih Kaabachi ${ }^{3}$, Eya Kalai ${ }^{1}$, Wided Smaoui ${ }^{4}$, Sonia Rekik ${ }^{5}$, \\ Asma Krir ${ }^{2}$, Nesrine Issaoui ${ }^{2}$, Kamel Hamzaoui ${ }^{3}$, Hela Sahli ${ }^{1}$, Elhem Cheour El Kateb ${ }^{1}$, \\ Mohammed Karim Zouaghi ${ }^{4}$, Afef Bahlous ${ }^{2}$ \\ ${ }^{1}$ Immuno-Rheumatology Research Laboratory, Rheumatology Department, La Rabta Hospital, \\ University of Tunis-El Manar, Tunis, Tunisia \\ ${ }^{2}$ Laboratory of Clinical Biochemistry and Hormonology; Pasteur Institute of Tunis, \\ University of Tunis-El Manar, Tunis, Tunisia \\ ${ }^{3}$ Department of Basic Sciences, Medicine School of Tunis, University of Tunis-El Manar, Tunis, Tunisia \\ ${ }^{4}$ Nephrology Department, La Rabta Hospital, Medicine School of Tunis, University of Tunis-El Manar, Tunis, Tunisia \\ ${ }^{5}$ Rheumatology Department, La Rabta Hospital, Medicine School of Tunis, University of Tunis-El Manar, Tunis, Tunisia
}

\section{Summary}

Background: Chronic kidney disease (CKD) is associated with numerous complications such as bone mineral disorder. The aim of our study was to analyze the correlation of bone turnover markers with Bone Mineral Density (BMD) measurements in Tunisian end stage renal diseases (ESRD) patients.

Methods: This study included 100 ESRD Tunisian patients. Their estimated glomerular filtration rate (eGFR) was $<15$ $\mathrm{mL} \times \min ^{-1} \times\left(1.73 \mathrm{~m}^{2}\right)^{-1}$, which requires hemodialysis. Bone-specific alkaline phosphatase (BALP) serum concentration was determined with a chemiluminescence immunoassay. Fibroblast Growth Factor 23 (FGF23) serum was assessed by Enzyme-Linked Immunosorbent Assay method. The serum levels of 25-Hydroxyvitamin D $(25(\mathrm{OH}) \mathrm{D})$, intact parathyroid hormone (iPTH) and Beta cross-laps (CTX) was measured by Electrochemiluminescence Technology. DEXA (dual-energy x-ray absorptiometry) technique was used to evaluate BMD.

Results: We observed a statistically significant negative correlation between BALP levels and total body BMD ( $r=$

\footnotetext{
Address for correspondence:

Mouna Bouksila

Immuno-Rheumatology Research Laboratory, Rheumatology Department, La Rabta Hospital, University of Tunis-El Manar, Tunis, Tunisia

Address: 13; Pasteur place, B.P. 74; 1002 Tunis, Belvedere, Tunisia

Phone number: +21695361654

e-mail:manou.bouk@live.fr
}

\section{Kratak sadržaj}

Uvod: Hronično bubrežno oboljenje (CKD) je praćeno brojnim komplikacijama kao što je poremećaj minerala kostiju. Svha ovog izučavanja je bila da analizira korelaciju markera promena u kostima sa mineralnom gustinom kostiju (BMD) i stupnja renalnih oboljenja kod pacijenata u Tunisu. Metode: Proučavanje je uključilo 100 ESRD tuniskih pacijenata. Njihova prosečna brzina glomerularne filtracije (eGFR) bila je $<15 \mathrm{~mL} \times \min ^{-1} \times\left(1,73 \mathrm{~m}^{2}\right)^{-1}$ što je zahtevalo hemodijalizu. Koštana alkalna fosfataza (BLAP) određena je u serumu hemilumiiscentnom imuno metodom. Faktor rasta fibroblasta 23 (FGF23) u serumu određen je enzimskom imunosorbetnom metodom. Nivoi 25-hidroksivitamina D $(25(\mathrm{OH}) \mathrm{D})$, intaktnog paratiroidnog hormona (iPTH) i betakros-lapsa (CTX) izmereni su elektrohemiluminiscentnom DEXA tehnologijom (dual-energy x-ray apsorpciometrijom) kako bi se procenila BMD.

Rezultati: Nađena je značajno negativna statistička korelacija BALP nivoa i ukupnog telesnog BMD $(r=-0,268$ : $P=0,015)$ $u$ femoralnom delu vrata (FN) $(r=-0,219 ; P=0,037)$. BALP je bila u negativnoj korelaciji sa ukupnom BMD

List of abbreviations: CKD, Chronic kidney diseases; ESRD, End stages renal diseases; 25(OH)D, 25-Hydroxyvitamin D; iPTH, intact parathyroid hormone; CTX, beta-cross-laps; BALP, bone alkaline phosphatase; FGF23, Fibroblast Growth Factor 23; PAL, alkaline phosphatase; DEXA, Dual-Energy X-ray Absorptiometry; BTM, bone turnover markers; LS-BMD, Lumbar Spine-Bone Mineral Density; LS-Z, Lumbar Spine-Z scores; LS-T, Lumbar Spine-T scores; FN-BMD, Femoral Neck-Bone Mineral Density; FN-T, Femoral Neck-T scores; FN-Z, Femoral Neck-Z scores; Total-BMD, Total-Bone Mineral Density; PTG, Parathyroid gland; eGFR, estimated glomerular filtration. 
-0.268; $P=0.015)$ particularly in femoral neck (FN) $(r=-$ $0.219 ; P=0.037)$. BALP concentrations were negatively associated with total BMD especially in $\mathrm{FN}$ for patients with $\mathrm{BMI}<30$, FGF23 concentrations were also negatively correlated with BMD in lumbar spine site (LS) $(r=-0.209$; $P=0.046$ ). For osteopenic patients we found an inverse correlation between 25(OH)D concentrations and BMD in LS position $(r=-0.336 ; P=0.038)$. In men group, we have also found a negative correlation between $\mathrm{iPTH}$ and total BMD ( $r=-0.326 ; P=0.015)$. However we found a positive correlation between calcium expression and BMD in LS site ( $r=0.270 ; P=0.031)$.

Conclusions: FGF23 and BALP can predict bone loss in ESRD through their strong correlation with BMD in LS and FN sites respectively.

Keywords: 25-Hydroxyvitamin D, bone alkaline phosphatase, bone mineral density, bone mineral disorder, chronic kidney disease, fibroblast growth factor 23

\section{Introduction}

New biochemical markers are being developed as non invasive exploration methods of bone turnover particularly in renal diseases (1). Chronic kidney diseases (CKD) is a complex renal pathology associated with numerous complications such as kidney failure and bone mineral disorder (CKD-MBD) (2). The alteration of kidney function is related to a progressive bone loss, leading to the onset of osteoporosis (3). The abnormalities in bone volume were positively correlated with CKD severity, especially during end stage renal disease (ESRD) (4-6). An overexpression of bone turnover markers (BTM) was also observed during CKD. These markers indicating bone remodeling include calcium; phosphate; $\mathrm{PTTH}$, alkaline phosphatase (PAL) and BALP (7-9).

In CKD PAL and BALP measurements were widely recommended, to evaluate not only in the bone mineral status disorder, but also in the rate of vascular calcification (10). BALP is an enzyme that promotes bone mineralization by inactivating pyrophosphate and osteopontin, which are both inhibitors of bone mineralization $(11,12)$. I's role is essential in determining the rate of bone formation and turnover (13). While BALP is a marker of bone formation, serum CTX, a collagen-degradation product, is a marker of bone resorption $(14,15)$.

FGF23, formed by osteocyte and osteoblast cells, is implicated in addition to BALP in bone remodeling $(13,16,17)$. In fact, FGF23 has been considered as a bone marker and has a crucial role, not only in mineral balance, but also in bone mineralization and vascular calcification $(18,19)$.

It's also a regulator of vitamin $D$ metabolism through a different bone-kidney axis. It inhibits renal tubular reabsorption of phosphate, impacting both calcium and phosphate bone transportation, and induces reduction of $1,25(\mathrm{OH}) 2 \mathrm{D}$ concentrations (20-23). naročito kod FN pacijenata sa BMI < 30, FGF23 koncentracije su bile takođe $u$ negativnoj korelaciji sa BMD u lumbalnom delu (LS) ( $r=-0,209, P=0,046)$. Kod osteopeničnih pacijenata nađene je inverzna korelacija između 25(OH)D koncentracije i DMD u LS polažaju $(r=-0,336$; $P=0,038)$. U grupi muških osoba nađena je negativna korelacija između iPTH i ukupnog BMD ( $r=-0,326 ; \mathrm{P}=0,015)$. Međutim, nađena je pozitivna korelacija između ekspresije kalcijuma i BMD u LS ( $r=0,270: P=0,031)$.

Zaključak: FGF23 i BALP mogu da predvide gubitak kosti kod ESRD preko izrazite korelacije sa BMD u LS i FN položajima.

Ključne reči: 25-hidroksivitamin $D$, koštana alkalna fosfataza, miniralna gustina kosti, hronično oboljenje bubrega, faktor rasta fibroblasta 23

As a result, the rise in $\mathrm{PTTH}$ can be attributed not only to the absence of the direct impact of $1,25(\mathrm{OH}) \mathrm{D}$ on parathyroid gland (PTG) and bone, but also to the intestinal absorption of calcium (24). Moreover, the decrease of serum 1-25-hydroxyvitamin $D$, commonly observed in CKD, may have an important role in the pathogenesis of osteoporosis in such patients (25).

Those markers can probably provide a better control of BMD to hemodialysis patients. In addition, the use of DEXA is recommended by multiple guidelines, thanks to its efficiency in discriminating bone loss in ESRD patients (8).

The aim of this study was to evaluate the correlation of bone turnover markers with DEXA measurements in Tunisian ESRD patients.

\section{Materials and Methods}

\section{Patients}

This study included 100 hemodialysis patients. A male predominance was found $(64 \%)$ with a sexratio of 1.77 . The mean age was $57.5 \pm 15.00$ years old; with extremes going from 23 to 94 years old and the mean disease duration was $7.20 \pm 4.20$ years. All patients were in ESRD. Before starting hemodialysis, their eGFR value was less than $<15 \mathrm{~mL} \times \min ^{-1} \times$ $\left(1.73 \mathrm{~m}^{2}\right)^{-1}$. Our population was classified into two major groups: diabetics $(\mathrm{N}=60)$ and non-diabetics $(\mathrm{N}=40)$.

Patients have not received vitamin D supplementation in the last six months. Their clinical and demographic data were gathered in Table I. They were followed-up at the Nephrology Department of the RABTA Hospital and they were undergoing hemodialysis three times a week during 4 to 5 hours. 
Exclusion criteria were patients with liver disease, cirrhosis, gastrectomy, parathyroidectomy, hysterectomy and evolutive neoplasias as well as those under glucocorticoides. Patients' demographic and medical information including age, sex, height, weight, smoking history, heart disease, hypertension, diabetes, hyperlipidemia and drug history were gathered and recorded in predefined data sheets.

This study was approved by the local ethics committee of the RABTA hospital, and a written informed consent was obtained from all the participants conformably to the ethical standards and the Declaration of Helsinki Principles.

\section{Human samples}

All blood samples were obtained after 8 hours an overnight fasting, just before starting hemodialysis. Subsequent plasma and serum were separated within 30 minutes and the samples were conserved frozen at $-80{ }^{\circ} \mathrm{C}$ until assays were realized.

\section{Serum markers}

Serum BALP was determined by chemiluminescence immunoassay (Access-Ostase ${ }^{\circledR}$ immunoassay, Beckman-Coulter Inc., USA.) on the ACCESS immunoassay system; ACCESS ${ }^{\circledR}$ Ostase is a chemiluminescent immunoassay with paramagnetic particles for the quantitative determination of serum BALP using the ACCESS Analyzer. The test is a onestep enzyme immunoassay. Whereas serum FGF23 was assessed by ELISA method (Demeditec ${ }^{\circledR}$, Germany). It's a Sandwich ELISA method, which measures the amount of antigen between two layers of antibodies. The antigen to be measured contain two antigenic sites capable of binding to antibody.

The following parameters were measured by Integra 400 Plus analyzer (Germany, Roche Diagnostics ${ }^{\circledR}$ ): Total serum calcium (endpoint method with 5-nitro-5'-methyl-BAPTA (NM-BAPTA), Creatinine (kinetic compensated Jaffe method); Phosphate (endpoint method with ammonium molybdate) and PAL (kinetic method with p-nitrophenylphosphate).

Serum 25(OH)D, iPTH and CTX were measured by Electrochemiluminescence Technology: (Cobas e411 analyzer, Germany, Roche Diagnostics ${ }^{\circledR}$ ). This method is an immunological test for a quantitative determination of $25(\mathrm{OH}) \mathrm{D}$, iPTH and CTX base on a competition principle for 25(OH)D and a "sandwich" for the two others assays. The inter-assay coefficient of variation for 25(OH)D, iPTH, CTX, BALP and FGF23 were lower than $11 \%, 4 \%, 9 \%, 6.5 \%$ and $12 \%$ respectively.

\section{Measurements for $B M D$}

Dual-Energy X-ray Absorptiometry technique was used for the evaluation of BMD and the detection of osteoporosis. DEXA measurements in lumbar spine and femoral neck were assessed using WHO (World Health Organization) criteria as a cutoff point. $B M D$ results were evaluated according to the T-score based on the number of standard deviations (SDs). The normal BMD is defined by a T-scores higher than -1, A T-score lower than -1 defines osteopenia, while osteoporosis is associated with values lower then $-2,5$ (26).

According to the WHO traditional densitometric classification, for DEXA measurements, when the studied population includes post-menopausal women or men aged $\geq 50$ years old; the T-score values show the type of bone loss. This diagnosis of WHO should also be applied to menopausal women (26). In postmenopausal women, the diagnosis of bone loss is made normal when the values of T-scores will be equal or exceed $-1,0$ SD. In men over the age of 50, osteopenia (low bone density) is defined by T-scores values between $-1,0$ and- 2,5 SDs. In menopausal transition osteoporosis is determined by values of $\mathrm{T}$ scores equal or lower than $-2,5$ SDs (27).

\section{Statistical analysis}

Means and SD were determined for the quantitative variables. Data was analyzed using SPSS software version 24 . The comparative study was realized using the Student's t-test for quantitative variables and the $\mathrm{X}^{2}$ test or the Fischer's exact test for qualitative variables. The correlation between parameters was evaluated by Pearson correlation analysis. The significance level $P$ was set at 0.05 .

\section{Results}

The means of $B M D$ in the lumbar spine, femoral neck and in the total body were respectively $1.14 \pm 0.22$ $\mathrm{g} / \mathrm{cm}^{2} ; 0.92 \pm 0.16 \mathrm{~g} / \mathrm{cm}^{2}$ and $1.11 \pm 0.12 \mathrm{~g} / \mathrm{cm}^{2}$, The means of $L S, F N, T$ and $Z$ score were respectively $-0.58 \pm 1.81 \mathrm{~g} / \mathrm{cm}^{2} ; 0.12 \pm 1.74 \mathrm{~g} / \mathrm{cm}^{2} ;-1.08 \pm$ $1.26 \mathrm{~g} / \mathrm{cm}^{2}$ and $-0.38 \pm 1.15 \mathrm{~g} / \mathrm{cm}^{2}$ (Table l).

For the lumbar spine T score, 58 patients (58\%) had normal bone profile, 29 (29\%) patients were osteopenic and 13 (13\%) were osteoporotic. As for the femoral neck T-score, 51 (51\%) had normal bone profile, $36(36 \%)$ patients were osteopenic and 13 (13\%) were osteoporotic (Table I). Twenty-two osteoporotic patients where identified (68\% female versus $32 \%$ male). Their mean age was $68.23 \pm$ 10.69 years old; higher than observed in normal profile patients ( $52.70 \pm 16.26$ years) and in osteopenic ones (56.34 \pm 12.82 years). Moreover, the duration of hemodialysis expressed in months was 
Table I Clinical and biological characteristics of hemodialysis patients.

\begin{tabular}{|c|c|}
\hline Patients Characteristic & Mean \pm SD (N \%) \\
\hline Population numbers & $N=100$ \\
\hline \multicolumn{2}{|l|}{ Biological parameters } \\
\hline Age (years) & $57.50 \pm 15.01$ \\
\hline Gender (Female /Male) & $36 / 64$ (36\% / 64\%) \\
\hline Tabacco & $48(48 \%)$ \\
\hline Alcohol & $19(19 \%)$ \\
\hline $\begin{array}{l}\text { Duration of dialysis } \\
\text { (months) }\end{array}$ & $79.91 \pm 51.13$ \\
\hline Weight $\left(\mathrm{m}^{2}\right)$ & $70.27 \pm 12.45$ \\
\hline Height $(\mathrm{kg})$ & $1.64 \pm 0.10$ \\
\hline $\mathrm{BMI}\left(\mathrm{kg} / \mathrm{m}^{2}\right)$ & $26.30 \pm 4.95$ \\
\hline \multicolumn{2}{|l|}{ Clinical parameters } \\
\hline CTX (ng/mL) & $1.99 \pm 1.40$ \\
\hline iPTH (pmol/L) & $32.50 \pm 33.42$ \\
\hline $25(\mathrm{OH}) \mathrm{D}(\mathrm{nmol} / \mathrm{L})$ & $37.37 \pm 25.45$ \\
\hline $\operatorname{BALP}(\mathrm{ng} / \mathrm{mL})$ & $24.31 \pm 19.04$ \\
\hline FGF23 (pg/mL) & $212.31 \pm 228.90$ \\
\hline Calcium (mmol/L) & $2.21 \pm 0.20$ \\
\hline Phosphate (mmol/L) & $1.96 \pm 1.06$ \\
\hline Creatinine $(\mu \mathrm{mol} / \mathrm{L})$ & $765.02 \pm 606.52$ \\
\hline PAL (UI/L) & $100.90 \pm 48.33$ \\
\hline \multicolumn{2}{|c|}{ Bone mineral density data [range] } \\
\hline LS-BMD $\left(\mathrm{g} / \mathrm{cm}^{2}\right)$ & $1.14 \pm 0.22[0.63-1.95]$ \\
\hline LS-T & $-0.58 \pm 1.8[1-4.6-6.1]$ \\
\hline LS-Z & $0.12 \pm 1.74[-3.8-7.2]$ \\
\hline FN-BMD $\left(\mathrm{g} / \mathrm{cm}^{2}\right)$ & $0.92 \pm 0.16[0.64-1.4]$ \\
\hline FN-T & $-1.08 \pm 1.26[-3.2-2.4]$ \\
\hline FN-Z & $-0.38 \pm 1.15[-2.2-3.3]$ \\
\hline TOTAL-BMD $\left(\mathrm{g} / \mathrm{cm}^{2}\right)$ & $1.11 \pm 0.12[0.86-1.41]$ \\
\hline LS-T $\quad$ LS-T $\geq-1$ & $58(58 \%)$ \\
\hline$-2.5<$ LS-T $<-1$ & $29(29 \%)$ \\
\hline LS-T $\quad-2.5$ & $13(13 \%)$ \\
\hline LS-T $\geq-1$ & $58(58 \%)$ \\
\hline FN-T LS-T $\geq-1$ & $51(51 \%)$ \\
\hline$-2.5<$ LS-T $<-1$ & $36(36 \%)$ \\
\hline LS-T $\quad-2.5$ & $13(13 \%)$ \\
\hline
\end{tabular}

BMI: Body Mass index; CTX: Beta cross laps; iPTH: parathyroid hormone; 25(OH)D:25-hydroxyvitamin D; FGF23: fibroblast growth factor 23; PAL: alkaline phosphatase; BALP: bone alkaline phosphatase, BMD: Bone Mineral Density; LS-BMD: Bone Mineral Density in Lumbar spine site; FN-BMD: Bone Mineral Density in Femoral neck; LS-T: T score in Lumbar spine site, FN-T: T score in Femoral neck; LS-Z: Z score in Lumbar spine site, FN-Z: Z score in Femoral neck longer in osteoporotic patients (102.45 \pm 60.42) compared to osteopenic patients (66.21 \pm 31.94) (Table II).

$B M D$ in $L S$ and $F N$ sites were significantly lower in women. Significant differences between male and female patients in BMD $\mathrm{T}$ score and $\mathrm{Z}$ score were observed $(P<0.05)$ both in $L S$ and $F N$ sites. The mean values of bone markers (iPTH, CTX, BALP and FGF23) were higher in female patients but not statistically significant $(P>0.05)$ (Table III).

There was a significant negative correlation between BALP concentrations and total body bone mineral density $(r=-0.268 ; P=0.015)$ particularly in the femoral neck ( $r=-0.219 ; P=0.037)$. Similarly, a significant negative correlation between FGF23 concentrations and the BMD in lumbar spine $[(r=-0.209 ; P=0,046$ and $T$ scores $(r=-0.213 ; P$ $=0.041)]$ was retained (Figure I).

Additionally, low 25(OH)D concentrations were negatively correlated with LS-Z in osteopenic patients ( $r=-0.336 ; P=0.038$ ) (Table IV). Moreover, in osteoporotic patients, we found that phosphate concentrations were negatively associated with FN-T $(r=-0.569 ; P=0.005) ; F N-B M D(r=-0,575 ; P=$ $0.005)$ and total BMD ( $r=-0.603 ; P=0.010)$.

Stratification based on different biological and clinical parameters revealed many different correlations. We observed a negative correlation between calcium concentrations and total BMD in women group $(r=-0.346 ; P=0.048)$. In men group, here was a negative correlation between $\mathrm{PTTH}$ and total BMD ( $r=-0.326 ; P=0.015)$. However, $a$ positive correlation was detected between both calcium and BMD in lumbar spine site ( $r=0.270$; $P$ $=0.031)$ and total BMD $(r=0.295 ; P=0.027)$ (Table IV).

In patients younger than 55 years old, we found that serum concentration of BALP is negatively correlated with FN-T ( $r=-0.386 ; P=0.018)$ and total BMD in FN ( $r=-0.349 ; P=0.034)$. In addition, a significant negative association between creatinine concentrations and Z-LS was detected ( $r=$ $-0.457 ; P=0.0027$ ). In patients older than 55 years, a negative correlation was found between iPTH and total BMD ( $r=-0.322 ; \mathrm{P}=0.020)$ (Table V).

Analysis based on BMI factor indicated that in patients with $\mathrm{BMI}$ lower than 30, there were positive correlations between 25(OH)D and FN-T, FN-Z and FN-BMD [ $r=0.367 ; P=0.015) ;(r=0.354 ; P=$ 0.019); $(r=0.355 ; P=0.019)]$ respectively. In patients with $\mathrm{BMI}<30$, BALP was negatively associated with FN-T ( $r=-0.319 ; P=0.042)$, FN-Z $(r=-0.337 ; P=0.031), F N-B M D(r=-0.311 ; P=$ $0.048)$ and Total BMD ( $r=-0.371 ; P=0,023)$. In this group, the $\mathrm{iPTH}$ concentrations were negatively correlated with total BMD ( $r=-0.375 ; P=0.018)$, while a positive significant association was found 
Table II Distribution of clinical characteristics of patients according to BMD.

\begin{tabular}{|c|c|c|c|}
\hline & $\begin{array}{c}\text { Normal } \\
\text { Mean } \pm \text { SD / N (\%) }\end{array}$ & $\begin{array}{c}\text { Osteopenia } \\
\text { Mean } \pm \text { SD / N (\%) }\end{array}$ & $\begin{array}{c}\text { Osteoporosis } \\
\text { Mean } \pm \text { SD / N (\%) }\end{array}$ \\
\hline $\operatorname{Sex}(M / F)$ & $30 / 10(75 \% / 25 \%)$ & $27 / 11(71 \% / 29 \%)$ & $7 / 15(32 \% / 68 \%)$ \\
\hline Age (years) & $52.70 \pm 16.26$ & $56.34 \pm 12.82$ & $68.23 \pm 10.69$ \\
\hline Tabacco & $22(55 \%)$ & $20(53 \%)$ & $6(27 \%)$ \\
\hline Alcohol & $9(23 \%)$ & $10(26 \%)$ & 0 \\
\hline Physical activity & $5(13 \%)$ & $5(13 \%)$ & $2(9 \%)$ \\
\hline Duration of Dialysis (months) & $80.53 \pm 56.86$ & $66.21 \pm 31.94$ & $102.45 \pm 60.42$ \\
\hline Weight (kg) & $71.19 \pm 10.57$ & $69.32 \pm 12.89$ & $70.23 \pm 15.09$ \\
\hline Height $\left(\mathrm{m}^{2}\right)$ & $1.68 \pm 0.09$ & $1.64 \pm 0.09$ & $1.57 \pm 0.09$ \\
\hline $\mathrm{BMI}\left(\mathrm{kg} / \mathrm{m}^{2}\right)$ & $25.40 \pm 3.78$ & $25.81 \pm 4.41$ & $28.79 \pm 6.81$ \\
\hline CTX (ng/mL) & $2.02 \pm 1.44$ & $2.05 \pm 1.44$ & $1.81 \pm 1.29$ \\
\hline iPTH (pmol/L) & $32.23 \pm 36.41$ & $34.38 \pm 30.40$ & $29.61 \pm 34.23$ \\
\hline $25(\mathrm{OH}) \mathrm{D}(\mathrm{nmol} / \mathrm{L})$ & $36.17 \pm 23.77$ & $37.42 \pm 25.55$ & $39.50 \pm 29.12$ \\
\hline $\operatorname{BALP}(\mathrm{ng} / \mathrm{mL})$ & $22.47 \pm 18.89$ & $21.84 \pm 11.95$ & $31.91 \pm 26.72$ \\
\hline FGF23 (pg/mL) & $171.38 \pm 186.52$ & $214.95 \pm 238.08$ & $300.60 \pm 281.06$ \\
\hline Calcium (mmol/L) & $2.21 \pm 0.16$ & $2.21 \pm 0.17$ & $2.25 \pm 0.29$ \\
\hline Phosphate (mmol/L) & $1.94 \pm 1.09$ & $1.99 \pm 1.08$ & $1.95 \pm 1.02$ \\
\hline Creatinine $(\mu \mathrm{mol} / \mathrm{L})$ & $728.253 \pm 269.006$ & $696.870 \pm 311.260$ & $949.610 \pm 1175.74$ \\
\hline PAL (UI/L) & $93.44 \pm 48.26$ & $113.76 \pm 45.58$ & $91.50 \pm 53.59$ \\
\hline
\end{tabular}

BMI: Body Mass index; CTX: Beta cross laps; iPTH: parathyroid hormone; 25(OH)D: 25-hydroxyvitamin D; FGF23: fibroblast growth factor 23; PAL: alkaline phosphatase; BALP: bone alkaline phosphatase

Table III Comparison of clinical and biological characteristics between male and female.

\begin{tabular}{|c|c|c|c|}
\hline & $\begin{array}{c}\text { Female }(\mathrm{N}=36) \\
\text { Mean } \pm \mathrm{SD}\end{array}$ & $\begin{array}{l}\text { Male }(\mathrm{N}=64) \\
\text { Mean } \pm \mathrm{SD}\end{array}$ & $P$ \\
\hline Age (years) & $60.08 \pm 16.36$ & $56.05 \pm 14.12$ & 0.198 \\
\hline Duration of dialysis (months) & $75.25 \pm 52.26$ & $82.53 \pm 50.71$ & 0.497 \\
\hline Weight $(\mathrm{kg})$ & $66.39 \pm 14.73$ & $72.45 \pm 10.47$ & 0.034 \\
\hline Height $\left(m^{2}\right)$ & $1.55 \pm 0.07$ & $1.69 \pm 0.08$ & $<0.001$ \\
\hline BMI $\left(\mathrm{kg} / \mathrm{m}^{2}\right)$ & $27.65 \pm 6.59$ & $25.55 \pm 3.58$ & 0.083 \\
\hline CTX (ng/mL) & $2.08 \pm 1.48$ & $1.93 \pm 1.36$ & 0.617 \\
\hline iPTH (pmol/L) & $30.97 \pm 29.53$ & $33.35 \pm 35.59$ & 0.736 \\
\hline $25(\mathrm{OH}) \mathrm{D}(\mathrm{nmol} / \mathrm{L})$ & $34.90 \pm 26.02$ & $38.77 \pm 25.22$ & 0.468 \\
\hline $\operatorname{BALP}(\mathrm{ng} / \mathrm{mL})$ & $28.65 \pm 21.86$ & $22.28 \pm 17.38$ & 0.138 \\
\hline FGF23 (pg/mL) & $248.05 \pm 254.36$ & $193.24 \pm 213.91$ & 0.276 \\
\hline Calcium (mmol/L) & $2.23 \pm 0.24$ & $2.20 \pm 0.16$ & 0.440 \\
\hline Phosphate (mmol/L) & $1.88 \pm 0.99$ & $2.01 \pm 1.10$ & 0.571 \\
\hline PAL (UI/L) & $99.03 \pm 50.74$ & $102.07 \pm 47.69$ & 0.842 \\
\hline Creatinine $(\mu \mathrm{mol} / \mathrm{L})$ & $861.20 \pm 937.411$ & $710.92 \pm 285.710$ & 0.236 \\
\hline LS-BMD $\left(\mathrm{g} / \mathrm{cm}^{2}\right)$ & $1.02 \pm 0.22$ & $1.20 \pm 0.19$ & $<0.001$ \\
\hline LS-T & $-1.32 \pm 1.85$ & $-0.16 \pm 1.65$ & 0.002 \\
\hline LS-Z & $-0.14 \pm 1.48$ & $0.27 \pm 1.86$ & 0.251 \\
\hline FN-BMD $\left(\mathrm{g} / \mathrm{cm}^{2}\right)$ & $0.85 \pm 0.16$ & $0.95 \pm 0.16$ & 0.002 \\
\hline FN-T & $-1.25 \pm 1.32$ & $-0.98 \pm 1.22$ & 0.291 \\
\hline $\mathrm{FN}-\mathrm{Z}$ & $-0.51 \pm 1.03$ & $-0.30 \pm 1.22$ & 0.387 \\
\hline Total-BMD $\left(\mathrm{g} / \mathrm{cm}^{2}\right)$ & $1.04 \pm 0.10$ & $1.15 \pm 0.11$ & $<0.001$ \\
\hline
\end{tabular}

BMI: Body Mass index; CTX: Beta cross laps; iPTH: parathyroid hormone; 25(OH)D:25-hydroxyvitamin D; FGF23: fibroblast growth factor 23; PAL: alkaline phosphatase; BALP: bone alkaline phosphatase, BMD: Bone Mineral Density; LS-BMD: Bone Mineral Density in Lumbar spine site; FN-BMD: Bone Mineral Density in Femoral neck; LS-T: T score in Lumbar spine site, FN-T: T score in Femoral neck; LS-Z: Z score in Lumbar spine site, FN-Z: Z score in Femoral neck; P: signification 
Table IV Correlation between biochemical parameters and the BMD according to gender.

\begin{tabular}{|c|c|c|c|c|c|c|c|c|c|c|c|c|c|c|c|}
\hline & & \multicolumn{7}{|c|}{ Women } & \multicolumn{7}{|c|}{ Male } \\
\hline & & LS-Z & LS-T & FN-T & $\mathrm{FN}-\mathrm{Z}$ & $\begin{array}{c}\text { LS- } \\
\text { BMD }\end{array}$ & $\begin{array}{l}\text { FN- } \\
\text { BMD }\end{array}$ & $\begin{array}{l}\text { Total- } \\
\text { BMD }\end{array}$ & LS-Z & LS-T & FN-T & $\mathrm{FN}-\mathrm{Z}$ & $\begin{array}{c}\text { LS- } \\
\text { BMD }\end{array}$ & $\begin{array}{l}\text { FN- } \\
\text { BMD }\end{array}$ & $\begin{array}{l}\text { Total- } \\
\text { BMD }\end{array}$ \\
\hline \multirow[t]{2}{*}{$25(\mathrm{OH}) \mathrm{D}$} & $r$ & 0.057 & 0.105 & 0.225 & 0.191 & 0.098 & 0.216 & 0.172 & -0.184 & -0.161 & 0.003 & 0.001 & -0.122 & 0.029 & -0.085 \\
\hline & $P$ & 0.741 & 542 & 187 & 0.266 & 0.567 & 0.205 & 0.339 & 0146 & 0.203 & 0.982 & 0.993 & 0.337 & 0.818 & 0.533 \\
\hline \multirow[t]{2}{*}{ Phosphate } & $r$ & 0.189 & 086 & .087 & -0.029 & 0.077 & -0.096 & .164 & 0.109 & .135 & 74 & 0.107 & -0.164 & 0.088 & -0.140 \\
\hline & $P$ & 0.270 & 619 & 0.614 & 0.866 & 655 & 0.577 & 0.361 & 0.391 & 0.288 & 0.561 & 0.401 & 0.194 & 0.488 & 0.304 \\
\hline \multirow[t]{2}{*}{ BALP } & $r$ & 0.021 & -0.085 & -0.153 & -0.046 & -0.091 & -0.154 & -0.237 & -0.069 & -0.137 & -0.177 & -0.153 & -0.123 & -0.197 & -0.221 \\
\hline & $P$ & 0.915 & 0.660 & 0.429 & 0.813 & 0.637 & 0.425 & 0.225 & 0.595 & 0.287 & 0.168 & 0.234 & 0.341 & 0.125 & 0.108 \\
\hline \multirow[t]{2}{*}{ iPTH } & $r$ & -0.123 & -0.084 & -0.010 & -0.028 & -0.086 & -0.014 & -0.238 & -0.075 & -0.083 & -0.161 & -0.183 & -0.101 & -0.165 & -0.326 \\
\hline & $P$ & 0.483 & 0.632 & 0.957 & 0.872 & 0.622 & 0.934 & 0.190 & 0.558 & 0.519 & 0.209 & 0.151 & 0.429 & 0.196 & $0.015^{*}$ \\
\hline \multirow[t]{2}{*}{ PAL } & $r$ & 1 & 012 & 52 & 32 & 0.010 & 0.167 & 0.350 & 209 & -0.219 & 51 & -0.166 & $-0,219$ & -0.168 & -0.177 \\
\hline & $P$ & 6 & 5 & 3 & 3 & 0 & 2 & 33 & 77 & 72 & 52 & 58 & 0.272 & 400 & 0.418 \\
\hline \multirow[t]{2}{*}{ FGF23 } & $r$ & 7 & 203 & 93 & -0.100 & 98 & 91 & 59 & 72 & 76 & 67 & 64 & 166 & -0.152 & -0.137 \\
\hline & $P$ & 0.233 & 0.265 & 0.614 & 0.584 & 0.277 & 0.621 & 0.371 & 0.189 & 0.178 & 0.201 & 0.210 & 0.203 & 0.244 & 0.327 \\
\hline \multirow[t]{2}{*}{ CTX } & $r$ & 0.114 & 0.165 & 0.164 & 0.136 & 0.159 & 0.154 & -0.041 & -0.008 & -0.104 & -0.126 & $-0,082$ & -0.122 & -0.139 & -0.257 \\
\hline & $P$ & 0.507 & 0.336 & 0.341 & 0.430 & 0.354 & 0.368 & 0.819 & 0.952 & 0.417 & 0.327 & 0.523 & 0,342 & 0,278 & 0.057 \\
\hline \multirow[t]{2}{*}{ Creatinine } & $r$ & -0.088 & 30 & 0.009 & 0.062 & -0.124 & 0.012 & -0.161 & -0.149 & -0.053 & 0.102 & -0.004 & -0.037 & 0.094 & 0.050 \\
\hline & $P$ & 0.609 & 0.450 & 0.960 & 0.718 & 0.472 & 0.945 & 0.369 & 0.241 & 0.678 & 0.421 & 0.974 & 0.770 & 0.459 & 0,715 \\
\hline \multirow[t]{2}{*}{ Calcium } & $r$ & 0.094 & -0.077 & -0.274 & -0.119 & -0.077 & -0.264 & -0.346 & 0.115 & 0.209 & 0.223 & 0.137 & 0.270 & 0.231 & 0.295 \\
\hline & $P$ & 0.587 & 0.653 & 0.106 & 0.488 & 0.653 & 0.120 & $0.048 *$ & 0.365 & 0.098 & 0.076 & 0.282 & $0.031^{*}$ & 0.066 & $0.027 *$ \\
\hline
\end{tabular}

CTX: Beta cross laps; iPTH: parathyroid hormone; 25(OH)D:25-hydroxyvitamin D; FGF23: fibroblast growth factor 23; PAL: alkaline phosphatase; BALP: bone alkaline phosphatase, BMD: Bone Mineral Density; LS-BMD: Bone Mineral Density in Lumbar spine site; FNBMD: Bone Mineral Density in Femoral neck; LS-T: T score in Lumbar spine site, FN-T: T score in Femoral neck; LS-Z: Z score in Lumbar spine site, FN-Z: Z score in Femoral neck; p: signification

* $P$ value $<0.05 ; r$ : Correlation coefficient

Table V Correlation between biochemical parameters and BMD according to patients age.

\begin{tabular}{|c|c|c|c|c|c|c|c|c|c|c|c|c|c|c|c|}
\hline & & \multicolumn{7}{|c|}{ Age $<55$ years } & \multicolumn{7}{|c|}{ Age $>55$ years } \\
\hline & & LS-Z & LS-T & FN-T & $\mathrm{FN}-\mathrm{Z}$ & $\begin{array}{l}\text { LS- } \\
\text { BMD }\end{array}$ & $\begin{array}{l}\text { FN- } \\
\text { BMD }\end{array}$ & $\begin{array}{c}\text { TOTAL } \\
\text { BMD }\end{array}$ & LS-Z & LS-T & FN-T & $\mathrm{FN}-\mathrm{Z}$ & $\begin{array}{l}\text { LS- } \\
\text { BMD }\end{array}$ & $\begin{array}{l}\text { FN- } \\
\text { BMD }\end{array}$ & $\begin{array}{l}\text { TOTAL } \\
\text { BMD }\end{array}$ \\
\hline \multirow[t]{2}{*}{$25(\mathrm{OH}) \mathrm{D}$} & $r$ & -0.046 & -0.088 & 0.109 & 140 & -0.086 & 0.105 & 0.001 & -0.128 & -0.005 & 0.049 & -0.001 & 0.041 & 0.092 & 0.085 \\
\hline & $\mathrm{P}$ & 0.777 & 0.586 & 0.497 & .381 & 0.594 & 0.513 & 0.998 & 0.333 & 0.968 & 0.713 & 0.995 & 0.757 & 0.489 & 0.550 \\
\hline \multirow[t]{2}{*}{ Phosphate } & $r$ & -0.125 & -0.122 & -0.019 & -0.056 & -0.126 & -0.032 & -0.186 & 0.074 & 0.002 & -0.229 & -0.134 & -0.013 & -0.203 & -0.107 \\
\hline & $\mathrm{P}$ & 0.435 & 0.448 & 0.904 & 0.727 & 0.432 & 0.841 & 0.270 & 0.577 & 0.990 & 0.081 & 0.310 & 0.923 & 0.124 & 0.449 \\
\hline \multirow[t]{2}{*}{ BALP } & $r$ & 0.010 & -0.106 & -0.386 & -0.250 & -0.104 & -0.349 & -0.310 & -0.107 & -0.223 & -0.056 & -0.038 & -0.223 & -0.158 & -0.253 \\
\hline & $P$ & 0.950 & 0.531 & $0.018^{*}$ & 0.136 & 0.540 & $0.034^{*}$ & 0.078 & 0.441 & 0.105 & 0.687 & 0.787 & 0.104 & 0.253 & 0.079 \\
\hline \multirow[t]{2}{*}{ iPTH } & $r$ & -0.139 & -0.053 & -0.149 & -0.214 & -0.032 & -0.110 & -0.206 & -0.039 & -0.092 & -0.118 & -0.082 & -0.117 & -0.143 & -0.322 \\
\hline & $\mathrm{P}$ & 5 & 0 & 0.352 & 8 & 0.844 & \begin{tabular}{|l|}
0.492 \\
\end{tabular} & 0.220 & 71 & 95 & 0.380 & 0.542 & 0.386 & 0.290 & $0.022^{*}$ \\
\hline \multirow[t]{2}{*}{ PAL } & $r$ & 3 & 7 & -0 & 70 & 53 & 1 & 8 & 67 & 2 & 8 & 46 & 39 & 99 & 1 \\
\hline & $P$ & 434 & 0.448 & 0.663 & 8 & 0.531 & 0 & 8 & 0.740 & 0.912 & 0.772 & 0.821 & 0.846 & 0.964 & 0.396 \\
\hline \multirow[t]{2}{*}{ FGF23 } & $r$ & -0.202 & -0.212 & -0.073 & -0.092 & -0.222 & -0.101 & -0.219 & -0.188 & -0.225 & -0.259 & -0.213 & -0.210 & -0.247 & -0.204 \\
\hline & $P$ & 0.224 & 0.202 & 0.661 & 0.583 & 0.180 & 0.545 & 0.206 & 0.172 & 0.102 & 0.058 & 0.122 & 0.127 & 0.071 & 0.163 \\
\hline \multirow[t]{2}{*}{ CTX } & $r$ & -0.053 & -0.015 & -0.074 & -0.116 & -0.005 & -0.069 & -0.201 & 0.111 & -0.034 & -0.073 & 0.061 & -0.063 & -0.125 & -0.245 \\
\hline & $P$ & 0.742 & 0.925 & 0.646 & 0.468 & 0.977 & 0.668 & 0.233 & 0.405 & 0.801 & 0.586 & 0.649 & 0.636 & 0.350 & 0.082 \\
\hline \multirow[t]{2}{*}{ Creatinine } & $r$ & -0.457 & -0.312 & -0.064 & -0.186 & -0.309 & -0.071 & -0.130 & -0.022 & -0.085 & 0.061 & 0.086 & -0.085 & 0.026 & -0.146 \\
\hline & $P$ & $0.002^{*}$ & 0.047 * & 0.690 & 0.243 & 0.049 & 0.657 & 0.444 & 0.867 & 0.523 & 0.648 & 0.515 & 0.520 & 0.843 & 0.303 \\
\hline \multirow[t]{2}{*}{ Calcium } & $r$ & 0.183 & 0.024 & -0.053 & -0.184 & 0.016 & -0.180 & -0.128 & 0.016 & 0.038 & 0.088 & 0.076 & 0.088 & 0.088 & 0.029 \\
\hline & $\mathrm{P}$ & 0.228 & 0.874 & 0.729 & 0.226 & 0.918 & 0.236 & 0.427 & 0.902 & 0.777 & 0.508 & 0.567 & 0.508 & 0.505 & 0.838 \\
\hline
\end{tabular}

BMI: Body Mass index; CTX: Beta cross laps; iPTH: parathyroid hormone; 25(OH)D:25-hydroxyvitamin D; FGF23: fibroblast growth factor 23; PAL: alkaline phosphatase; BALP: bone alkaline phosphatase, BMD: Bone Mineral Density; LS-BMD: Bone Mineral Density in Lumbar spine site; FN-BMD: Bone Mineral Density in Femoral neck; LS-T: T score in Lumbar spine site, FN-T: T score in Femoral neck; LS-Z: Z score in Lumbar spine site, FN-Z: Z score in Femoral neck; p: signification

* $P$ value $<0,05 ; r$ : Correlation coefficient 


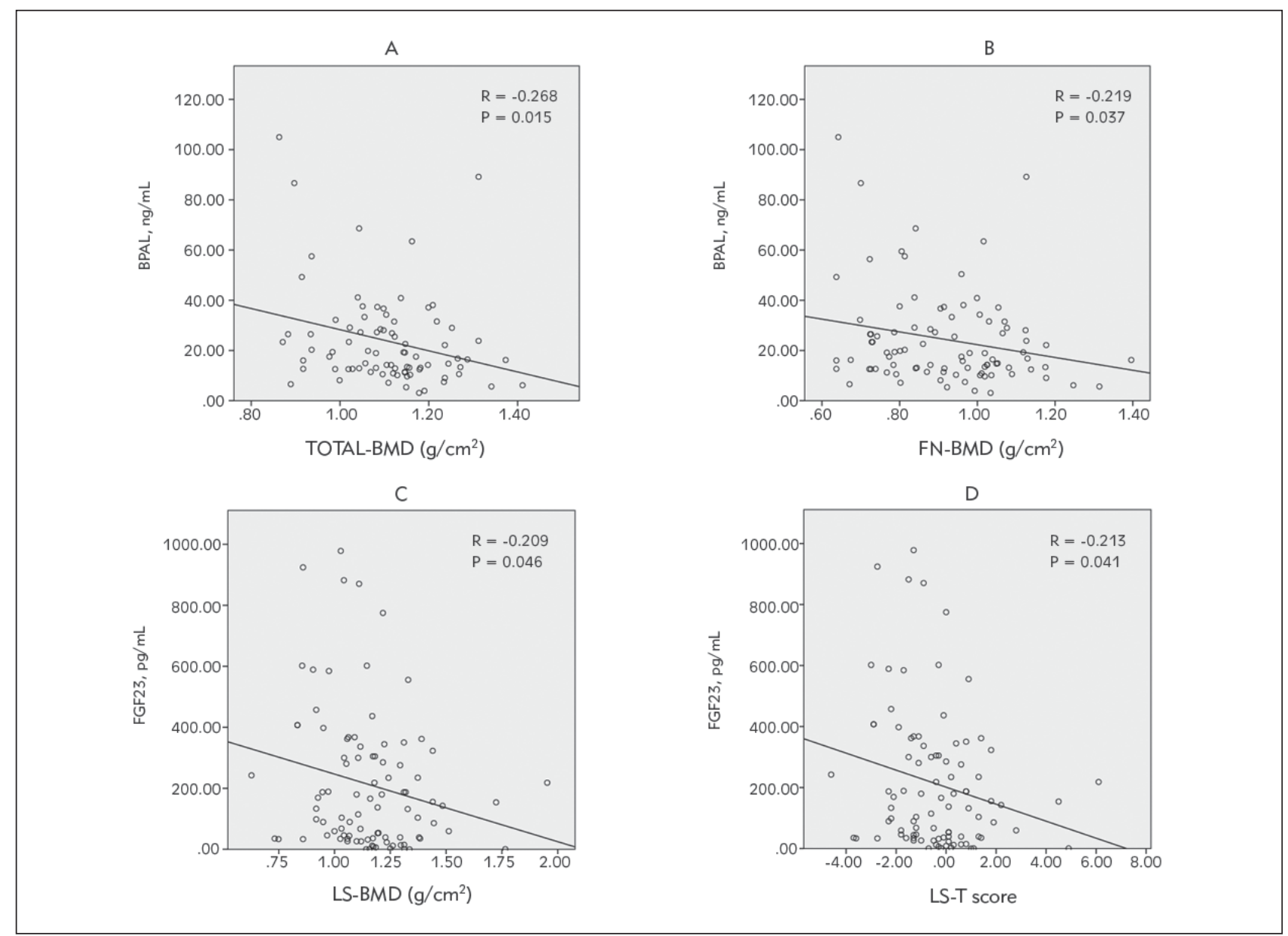

Figure 1 Correlation between BALP, FGF23 levels and BMD in hemodialysis patients A: Correlation between BALP levels and total BMD in hemodialysis patients; $B$ : Correlation between BALP and BMD in FN in hemodialysis patients; C: Correlation between FGF23 levels and LS-BMD in hemodialysis patients; D: Correlation between FGF23 levels and LS-T scores in hemodialysis patients

between creatinine and FN-BMD $(r=0.305 ; P=$ 0.046). Besides we detected a positive correlation between calcium concentrations and FN-T $(r=$ $0.302 ; \mathrm{P}=0.049$ ). In obese patients with a $\mathrm{BMI}$ higher than 30, a negative correlation was found between 25(OH)D and LS-Z $(r=-0.278 ; P=0.036)$.

\section{Discussion}

In our study, we found that serum BALP and FGF23 concentrations were associated with a loss of $B M D$ in femur and spine regions, respectively. Actually, in our research total body BMD measurements were negatively correlated with elevated BALP concentrations in Tunisian population. This was confirmed in many previous studies $(9,10,28)$. Furthermore, we observed a significant negative correlation between an increased BALP concentration and the $B M D$ only in the femoral neck site. Our findings were similar to those reported by Fidan et al. (7) in Turkey. However, many studies revealed that BALP was a predictive factor of bone loss, mainly in cortical sites, and was weakly associated with BMD essentially in trabecular sites $(9,29)$. These contradictory results could be explained by the difference between of studied populations, methods of analysis of bone loss measurements and the wide choice of measurement sites established by researchers. This negative correlation is affected by gender and age factors; Particularly old patients. But, lower BMI has no impact on this correlation when compared to overweight patient groups. Otherwise, serum BALP is the most sensitive and specific bone marker in the detection of bone turnover disorder in Tunisian patients with CKD and probably for patient with low BMI and younger than 55 years old. Besides, no correlation was established between PAL and total BMD neither in our results nor in others' $(30,31)$. Nevertheless, other studies showed that PAL concentrations were inversely correlated to the total $\operatorname{BMD}(32,33)$.

We also investigated the expression of FGF23 in Tunisian ESRD patients, as a bone marker that has a major role in bone turnover, and it is enclosed in bone mineral status $(20,21)$. We found that the increased 
concentrations of FGF23 were correlated with BMD loss in the lumbar spine site. However, this correlation is affected by age, sex and BMI. This result was partly confirmed by Malluche et al. (10) who correlated the rise of FGF23 concentration with BMD loss in spine not only at baseline but also after 12 months of follow-up.

In addition, and according to several studies, the low $25(\mathrm{OH}) \mathrm{D}$ concentration was one of the factors leading to osteoporosis not only in healthy persons but also in patients with CKD. This marker can be used for a primary detection of this bone pathology $(25,34)$. The decrease in bone mineralization and the reduction in calcium absorption is also one of the causes of this pathology.

In ESRD Tunisian patients, osteopenia status is related to low bone mass and reduced $25(\mathrm{OH}) \mathrm{D}$ levels. Thus, a vitamin D supplementation is needed. Alfacalcidol, Calcitriol, Paricalcitol and other vitamin $D$ analogues should be recommended to prevent the risk of osteoporosis (35). Furthermore, we found a negative correlation between iPTH concentrations and bone loss in various BMD measurement sites, in particular for men or old and overweight ESRD patients. However, our results were different from those reported in other studies $(3,7,36)$.

Our findings revealed the absence of significant correlation between the elevation of CTX concentrations and BMD in both the femur and the spine

\section{References}

1. Belino C, Meng C, Pereira L, Carvalho C, Neto R. The role of bone biomarkers and new imaging techniques in the management of patients with CKD-MBD. Port J Nephrol Hypert 2017; 31: 293-9.

2. Isakova T, Nickolas TL, Denburg M, Yarlagadda S, Weiner DE, Gutierrez OM, et al. KDOQI US Commentary on the 2017 KDIGO Clinical Practice Guideline Update for the Diagnosis, Evaluation, Prevention, and Treatment of Chronic Kidney Disease-Mineral and Bone Disorder (CKD-MBD). Am J Kidney Dis 2017; 70: 737-51.

3. Stefanović A, Ristovski-Kornic D, Kotur-Stevuljević J, Spasojević-Kalimanovska V, Vekić J, Miljković M, Paripović $D$, Peco-Antic A, Jelić-lvanović $Z$, Zeljković $A$. Alterations of $\mathrm{HDL}$ particles in children with end-stage renal disease. J Med Biochem 2017; 36: 358-65.

4. Jamal SA, Swan VJ, Brown JP, Hanley DA, Prior JC, Papaioannou $A$, et al. Kidney function and rate of bone loss at the hip and spine: the Canadian Multicentre Osteoporosis Study. Am J Kidney Dis 2010; 55: 291-9.

5. Malluche HH, Monier-Faugere MC. Renal osteodystrophy: what's in a name? Presentation of a clinically useful new model to interpret bone histologic findings. Clin Nephrol 2006; 65: 235-42.

6. Arslan DF, Karakoyun I, Basok IB, Aksit ZM, Celik E, Dogan K, Duman C. The Effects of Education and Training Given to Phlebotomists for Reducing Preanalytical Errors. J Med Biochem 2018; 37: 172-80. regions. In fact, the higher expression of CTX levels is caused by the decline of renal activity (37). Thus, no relationship between CTX and BMD measurements in hemodialysis was reported though their major implication in vascular calcification, and on secondary hyperparathyroidism $(38,39)$.

In conclusion, FGF23 and BALP can predict bone loss in end stages of CKD through their strong correlation with the lumbar spine and femoral neck sites respectively.

Acknowledgments: The authors would like to thank all the collaborators who supported our work and helped us gather better quality results in particular Mrs Anne Marie Marty.

Human and Animal Rights: The study was approved by the local ethics committee of the RABTA hospital, Tunisia.

Informed Consent: a written informed consent was obtained from all the participants conformably to the ethical standards and the Declaration of Helsinki Principles.

\section{Conflict of interest statement}

The authors stated that they have no conflicts of interest regarding the publication of this article.

7. Fidan N, Inci A, Coban M, Ulman C, Kursat S. Bone mineral density and biochemical markers of bone metabolism in predialysis patients with chronic kidney disease. J Investig Med 2016; 64: 861-6.

8. Nickolas TL, Cremers S, Zhang A, Thomas V, Stein E, Cohen $A$, et al. Discriminants of prevalent fractures in chronic kidney disease. J Am Soc Nephrol 2011; 22: 1560-72.

9. Nickolas TL, Stein EM, Dworakowski E, Nishiyama KK, Komandah-Kosseh M, Zhang CA, et al. Rapid cortical bone loss in patients with chronic kidney disease. J Bone Miner Res 2013; 28: 1811-20.

10. Baralić M, Brković V, Stojanov V, Stanković S, Lalić N, Đurić $P$, Đukanović $L j$, Kašiković $M$, Petrović $M$, Petrović $M$, Stošović $M$, Ležaić $V$. Dual roles of the mineral metabolism disorders biomarkers in prevalent hemodilysis patients: in renal bone disease and in vascular calcification. J Med Biochem 2019; 38: DOI: 10.2478/jomb-2018-0026.

11. Buchet R, Millan JL, Magne D. Multisystemic functions of alkaline phosphatases. Methods Mol Biol 2013; 1053: 27-51.

12. Johnson KA, Hessle L, Vaingankar S, Wennberg C, Mauro S, Narisawa S, et al. Osteoblast tissue-nonspecific alkaline phosphatase antagonizes and regulates PC-1. Am J Physiol Regul Integr Comp Physiol 2000; 279: R1365-77. 
13. Vervloet MG, Brandenburg VM. Circulating markers of bone turnover. J Nephrol 2017; 30: 663-70.

14. Pandrc SM, Ristić A, Kostovski V, Stanković M, Antić V, Milin-Lazović J, Ćirić J. The effect of early substitution of subclinical hypothyroidism on biochemical blood parameters and the quality of life. J Med Biochem 2017; 36: 127-36.

15. Baim S, Miller PD. Assessing the clinical utility of serum CTX in postmenopausal osteoporosis and its use in predicting risk of osteonecrosis of the jaw. J Bone Miner Res 2009; 24: 561-74.

16. Lu Y, Feng JQ. FGF23 in skeletal modeling and remodeling. Curr Osteoporos Rep 2011; 9: 103.

17. Bonewald LF, Wacker MJ. FGF23 production by osteocytes. Pediatr Nephrol 2013; 28: 563-8.

18. Guo Y-C, Yuan Q. Fibroblast growth factor 23 and bone mineralisation. Int J Oral Sci 2015; 7: 8.

19. Liu S, Guo R, Simpson LG, Xiao ZS, Burnham CE, Quarles LD. Regulation of fibroblastic growth factor 23 expression but not degradation by PHEX. J Biol Chem 2003; 278: 37419-26.

20. Feng JQ, Ward LM, Liu S, Lu Y, Xie Y, Yuan B, et al. Loss of DMP1 causes rickets and osteomalacia and identifies a role for osteocytes in mineral metabolism. Nat Genet 2006; 38: 1310.

21. Liu S, Zhou J, Tang W, Jiang X, Rowe DW, Quarles LD. Pathogenic role of Fgf23 in Hyp mice. Am J Physiol Endocrinol Metab 2006; 291: E38-E49.

22. Rowe P, Kumagai Y, Gutierrez G, Garrett I, Blacher R, Rosen $D$, et al. MEPE has the properties of an osteoblastic phosphatonin and minhibin. Bone 2004; 34: 303-19.

23. Liu S, Rowe PS, Vierthaler L, Zhou J, Quarles LD. Phosphorylated acidic serine-aspartate-rich MEPEassociated motif peptide from matrix extracellular phosphoglycoprotein inhibits phosphate regulating gene with homologies to endopeptidases on the X-chromosome enzyme activity. J Endocrinol 2007; 192: 261-7.

24. Quarles LD. Role of FGF23 in vitamin D and phosphate metabolism: implications in chronic kidney disease. Exp Cell Res 2012; 318: 1040-8.

25. Kiani A, Abedini A, Adcock IM, Mirenayat MS, Taghavi K, Mortaz E, Kazempour-Dizaji M. Association between vitamin $D$ deficiencies in sarcoidosis with disease activity, course of disease and stages of lung involvements. J Med Biochem 2018; 37: 103-9.

26. Messina C, Sconfienza LM, Bandirali M, Guglielmi G, Ulivieri FM. Adult Dual-Energy X-ray Absorptiometry in Clinical Practice: How I Report it. Semin Musculoskelet Radiol 2016; 20: 246-53.

27. Consensus development conference: diagnosis, prophylaxis, and treatment of osteoporosis. Am J Med 1993; 94: 646-50.

28. Nakashima A, Yorioka N, Doi S, Takasugi N, Shigemoto $\mathrm{K}$, Kohno N. Osteoprotegerin and bone mineral density in hemodialysis patients. Osteoporos Int 2006; 17: 8416.

29. Ueda $M$, Inaba $M$, Okuno S, Maeno $Y$, Ishimura $E$, Yamakawa $T$, et al. Serum BAP as the clinically useful marker for predicting BMD reduction in diabetic hemodialysis patients with low PTH. Life Sci 2005; 77: 1130-9.

30. Urena P, Hruby M, Ferreira A, Ang KS, de Vernejoul MC. Plasma total versus bone alkaline phosphatase as markers of bone turnover in hemodialysis patients. J Am Soc Nephrol 1996; 7: 506-12.

31. Bervoets AR, Spasovski GB, Behets GJ, Dams G, Polenakovic $M H$, Zafirovska $K$, et al. Useful biochemical markers for diagnosing renal osteodystrophy in predialysis end-stage renal failure patients. Am J Kidney Dis 2003; 41: 997-1007.

32. Park JC, Kovesdy CP, Duong U, Streja E, Rambod M, Nissenson AR, et al. Association of serum alkaline phosphatase and bone mineral density in maintenance hemodialysis patients. Hemodial Int 2010; 14: 182-92.

33. Bergman A, Qureshi AR, Haarhaus $M$, Lindholm B, Barany $\mathrm{P}$, Heimburger $\mathrm{O}$, et al. Total and bone-specific alkaline phosphatase are associated with bone mineral density over time in end-stage renal disease patients starting dialysis. J Nephrol 2017; 30: 255--62.

34. Li ZX, Xu C, Li YC, Sun QM. Osteoporosis biomarkers act as predictors for diagnosis of chronic renal insufficiency in elder patients. Int J Clin Exp Med 2015; 8: 5949.

35. Shroff R, Wan M, Nagler EV, Bakkalo lu S, Cozzolino M, Bacchetta J, et al. Clinical practice recommendations for treatment with active vitamin $D$ analogues in children with chronic kidney disease Stages $2-5$ and on dialysis. Nephrol Dial Transplant 2017; 32: 1114-27.

36. Ersoy FF, Passadakis SP, Tam P, Memmos ED, Katopodis PK, Özener Ç, et al. Bone mineral density and its correlation with clinical and laboratory factors in chronic peritoneal dialysis patients. J Bone Miner Metab 2006; 24: 79-86.

37. Pagani F, Bonetti G, Stefini F, Panteghini M. Evaluation of a fully automated assay to measure C-telopeptide of type I collagen in serum. Clin Chem Lab Med 2000; 38: 1111-3.

38. Ishimura E, Okuno S, Okazaki H, Norimine K, Yamakawa K, Yamakawa T, et al. Significant association between bone-specific alkaline phosphatase and vascular calcification of the hand arteries in male hemodialysis patients. Kidney Blood Press Res 2014; 39: 299-307.

39. Ma L, Zhao S, Li Z. Effects of parathyroidectomy on bone metabolism in haemodialysis patients with secondary hyperparathyroidism. Scand J Clin Lab Invest 2017; 77: 527-34. 